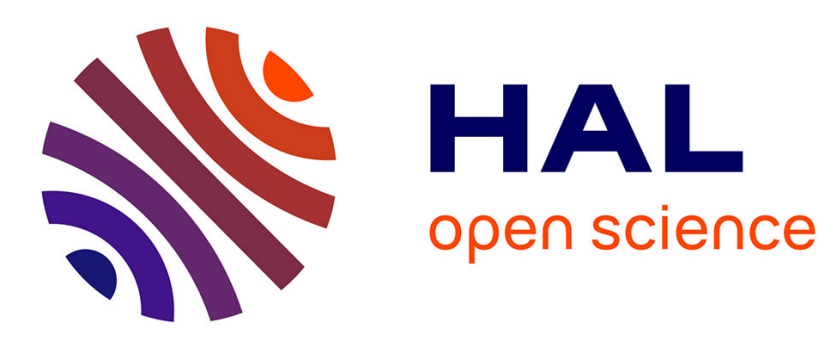

\title{
Magnetic Field Created by Tile Permanent Magnets
}

Romain Ravaud, Guy Lemarquand, Valérie Lemarquand

\section{To cite this version:}

Romain Ravaud, Guy Lemarquand, Valérie Lemarquand. Magnetic Field Created by Tile Permanent Magnets. IEEE Transactions on Magnetics, 2009, 45 (7), pp.2920-2926. 10.1109/TMAG.2009.2014752 . hal-00401733

\section{HAL Id: hal-00401733 https://hal.science/hal-00401733}

Submitted on 6 Jul 2009

HAL is a multi-disciplinary open access archive for the deposit and dissemination of scientific research documents, whether they are published or not. The documents may come from teaching and research institutions in France or abroad, or from public or private research centers.
L'archive ouverte pluridisciplinaire HAL, est destinée au dépôt et à la diffusion de documents scientifiques de niveau recherche, publiés ou non, émanant des établissements d'enseignement et de recherche français ou étrangers, des laboratoires publics ou privés. 


\title{
Magnetic Field Created by Tile Permanent
}

\section{Magnets}

R. Ravaud, G. Lemarquand, V. Lemarquand

\begin{abstract}
This paper presents the analytical calculation of the three components of the magnetic field created by tile permanent magnets whose magnetization is either radial or axial. The calculations are based on the coulombian model of permanent magnets. The magnetic field is directly calculated, without the magnetic potential. Both axial and radial magnetization of the tiles are considered. The expressions obtained give the magnetic field in all the space. Such analytical expressions are very useful for the design and optimization of many industrial applications.
\end{abstract}

\section{Index Terms}

Analytical model, magnetic field, permanent magnet, axial magnetization, radial magnetization

\section{INTRODUCTION} FTER the parallelepiped, the most common shape for a permanent magnet in electrical engineering is certainly the tile, which can also be described as a ring sector. Such tiles can be either axially or radially magnetized. Therefore, the calculation of the magnetic field created by tile magnets is of great utility and numerous approaches exist.

Numerical approaches do not allow one to perform numerous parametric studies quickly and have generally a high computational cost. Consequently, authors are looking for alternative solutions. Their approaches are often semianalytical ones [1], and they represent important steps toward the ideal analytical 
ones. In fact, different points of view can be adopted, which all look for the same thing: the calculation of the magnetic field and the magnetic forces. Kim et al. [2][3] calculate the magnetic force from the vector potential, with complete elliptic integrals and a mesh-matrix method. Kwon et al.[4] work with spherical coordinates and multipole expansions to calculate the far field of permanent magnet motors. Selvaggi et al.[5] propose an approach with cylindrical coordinates and the use of Green's function and Fourier series expansion to characterize the field from a set of permanent magnets. Babic and Akyel [6]-[8] calculate the force between coils thanks to Heuman's Lambda function. Toroidal harmonics yield interesting solutions to formulate the magnetic field created by permanent magnet cylinders [9]. Conway uses Bessel's function to calculate the inductance of coils using the vector potential [10] and proposes also a direct calculation of the magnetic field -without the vector potential- to reach the same goal [11].

While these studies show variety in the starting point for the problem they want to solve, many others are more specific and describe methods to calculate the magnetic field created by toroidal magnets. Perigo et al.[12] present analytical-integral expressions for the axial and radial magnetic flux density components of axially magnetized toroidal magnets, either on- or off-axis. The application considered is an electron beam focusing system. Zhilichev [13] works with cylindrical coordinates and uses separation of variables to evaluate the magnetic field from the scalar potential for tubular linear permanent magnet machines. He gives $2 \mathrm{D}$ and 3D approximations. Furlani et al. propose solutions based on the vector potential for radially polarized multipole cylindrical magnets [14] as well as for axially polarized magnets for axial field motors [15][16]. Rakotoarison et al.[17] give a semianalytical method to calculate the field created by radially magnetized tile magnets from the scalar potential. They use a coulombian model of the magnets and take the volume charge density into account.

All the studies dedicated to the calculation of the magnetic field created by toroidal or tile magnets are very useful: they constitute tools for the design and the optimization of devices which use such magnets and enable to meet specific requirements. For example, tile magnets with rotating magnetizations are used to make discrete Halbach cylinders [18] which have various applications, such as electrodynamic wheels [19] for maglev devices, the creation of homogeneous fields or tailored gradient fields [20], the magnetization of brushless machines to obtain sinusoidal field variation in the airgap [21]. Tile magnets are used with peculiar profiles to reduce the cogging torque in axial flux machines [22] as well as in radial 
46 flux ones [23], but also to control the torque in permanent magnet couplings [24][25] and gears. They 47 are constitutive parts of displacement sensors [26] and also ironless loudspeakers [27][28] demonstrating 48 their widespread use.

This paper presents analytical expressions for the three components of the magnetic field created by tile permanent magnets. The magnetic field is directly calculated, without a previous calculation of the section below and the radial magnetization in the second.

\section{Axially magnetized tiles}

\section{A. Notation and Geometry}

The geometry which is considered is a tile permanent magnet and the related parameters are shown

65 faces.

66 Let us consider a point $P$ on the ring sector upper face. The magnetic field $\vec{H}$ created by the source 67 point $P\left(r, \theta_{s}, z\right)$ at any observation point $M(r, \theta, z)$ of the space is given by (1).

$$
\vec{H}(r, \theta, z)=\frac{\sigma^{*}}{4 \pi \mu_{0}} \int_{\theta_{1}}^{\theta_{2}} \int_{r_{\text {in }}}^{r_{\text {out }}} \frac{\overrightarrow{P M}}{|\overrightarrow{P M}|^{3}} r_{1} d r_{1} d \theta_{s}
$$

68 where $\mu_{0}$ is the vacuum magnetic permeability $\left(\mu_{0}=4 \pi \cdot 10^{-7} S I\right)$ and $\sigma^{*}$ is the fictitious magnetic pole 


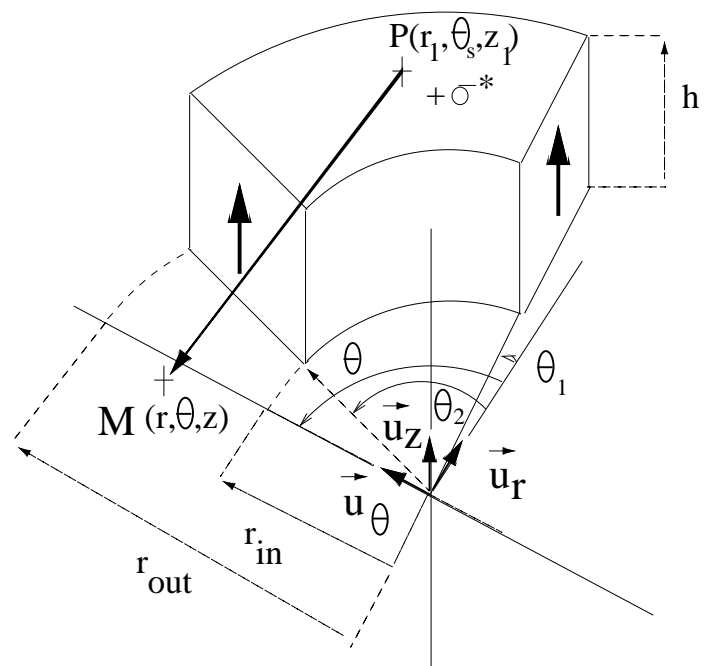

Fig. 1. Axially magnetized tile permanent magnet : parameter definition.

surface density in tesla. Equation (1) can be written as follows:

$$
\vec{H}(r, \theta, z)=\frac{\sigma^{*}}{4 \pi \mu_{0}} \int_{\theta_{1}}^{\theta_{2}} \int_{r_{\text {in }}}^{r_{\text {out }}} \frac{\left(r-r_{1} \cos \left(\theta-\theta_{s}\right)\right) \vec{u}_{r}-r_{1} \sin \left(\theta_{s}-\theta\right) \vec{u}_{\theta}+\left(z-z_{1}\right) \vec{u}_{z}}{\left(r_{1}^{2}+r^{2}+\left(z-z_{1}\right)^{2}-2 r_{1} r \cos \left(\theta-\theta_{s}\right)\right)^{\frac{3}{2}}} r_{1} d \theta_{s}
$$

B. Components along the three directions $\vec{u}_{r}, \vec{u}_{\theta}, \vec{u}_{z}$

The integration of (2) leads to the magnetic field components along the three axes defined $H_{r}(r, \theta, z)$,

$72 H_{\theta}(r, \theta, z), H_{z}(r, \theta, z)$.

73 1) Azimuthal component $H_{\theta}(r, \theta, z)$ : The magnetic field azimuthal component $H_{\theta}(r, \theta, z)$ created by 74 the upper face is given by (3).

$$
H_{\theta}(r, \theta, z)=\frac{\sigma^{*}}{4 \pi \mu_{0}}\left(\eta\left(\theta, \theta_{1}\right)-\eta\left(\theta, \theta_{2}\right)\right)
$$

75 with

$$
\begin{aligned}
\eta\left(\theta, \theta_{i}\right)= & \frac{\sqrt{r^{2}+r_{\text {in }}^{2}+(z-h)^{2}-2 r r_{\text {in }} \cos \left(\theta_{i}-\theta\right)}}{r} \\
& +\cos \left(\theta_{i}-\theta\right) \log \left[r_{\text {in }}-r \cos \left(\theta_{i}-\theta\right)+\sqrt{r^{2}+r_{\text {in }}^{2}+(z-h)^{2}-2 r r_{\text {in }} \cos \left(\theta_{i}-\theta\right)}\right. \\
& -\frac{\sqrt{r^{2}+r_{\text {out }}^{2}+(z-h)^{2}-2 r r_{\text {out }} \cos \left(\theta_{i}-\theta\right)}}{r}
\end{aligned}
$$




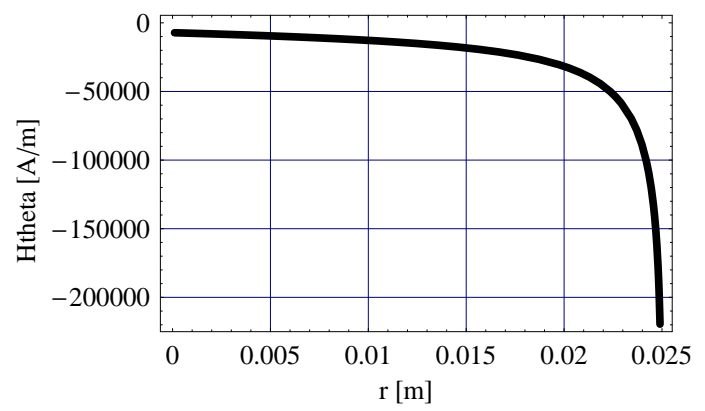

Fig. 2. Field azimuthal component $H_{\theta}(r, \theta, z)$ versus the radial distance $r$ of the observation point; $h=3 m m, r_{i n}=25 m m$, $r_{\text {out }}=28 m m, \theta_{1}=0, \theta_{2}=\frac{\pi}{2}, \theta=\frac{\pi}{2}$

$$
-\cos \left(\theta_{i}-\theta\right) \log \left[r_{\text {out }}-r \cos \left(\theta_{i}-\theta\right)+\sqrt{r^{2}+r_{\text {out }}^{2}+(z-h)^{2}-2 r r_{\text {out }} \cos \left(\theta_{i}-\theta\right)}\right]
$$

Equation (3) is valid for any observation point $M(r, \theta, z)$ with $0 \leq \theta \leq 2 \pi$. Figure 2 represents the azimuthal component $H_{\theta}(r, \theta, z)$ versus the radial distance $r$ of the obervation point. The parameter values are $h=3 \mathrm{~mm}, \theta_{1}=0 \mathrm{rad}, \theta_{2}=\frac{\pi}{2} \mathrm{rad}, \theta=0 \mathrm{rad}, r_{\text {in }}=25 \mathrm{~mm}, r_{\text {out }}=28 \mathrm{~mm}$.

2) Radial component $H_{r}(r, \theta, z)$ : The field radial component $H_{r}(r, \theta, z)$ created by the upper face is given by (5).

$$
H_{r}(r, \theta, z)=\frac{\sigma^{*}}{4 \pi \mu_{0}}\left(\alpha\left(u_{2}, r, z\right)-\alpha\left(u_{1}, r, z\right)\right)
$$

81 where

$$
u_{i}=\cos \left(\theta-\theta_{i}\right)
$$

82 and

$$
\begin{aligned}
\alpha\left(u_{i}, r, z\right)= & F_{1}\left(u_{i}, r, z\right)\left(G_{1}\left(u_{i}, r, z\right) \mathbf{E}^{*}\left[\arcsin \left[\frac{\sqrt{r^{2}+r_{\text {out }}^{2}-2 r r_{\text {out }} u_{i}+(z-h)^{2}}}{\left(r+r_{\text {out }}\right)^{2}+(z-h)^{2}}\right], \frac{\left(r+r_{\text {out }}\right)^{2}+(z-h)^{2}}{\left(r-r_{\text {out }}\right)^{2}+(z-h)^{2}}\right]\right) \\
& +F_{1}\left(u_{i}, r, z\right) \Pi^{*}\left[\arcsin \left[\frac{\sqrt{r^{2}+r_{\text {out }}^{2}-2 r r_{\text {out }} u_{i}+(z-h)^{2}}}{\left(r+r_{\text {out }}\right)^{2}+(z-h)^{2}}\right], \frac{\left(r+r_{\text {out }}\right)^{2}+(z-h)^{2}}{\left(r-r_{\text {out }}\right)^{2}+(z-h)^{2}}\right] \\
& -F_{2}\left(u_{i}, r, z\right)\left(G_{2}\left(u_{i}, r, z\right) \mathbf{E}^{*}\left[\arcsin \left[\frac{\sqrt{r^{2}+r_{\text {in }}^{2}-2 r r_{\text {in }} u_{i}+(z-h)^{2}}}{\left(r+r_{\text {in }}\right)^{2}+(z-h)^{2}}\right], \frac{\left(r+r_{\text {in }}\right)^{2}+(z-h)^{2}}{\left(r-r_{\text {in }}\right)^{2}+(z-h)^{2}}\right]\right)
\end{aligned}
$$




$$
\begin{aligned}
& -F_{2}\left(u_{i}, r, z\right) \Pi^{*}\left[\arcsin \left[\frac{\sqrt{r^{2}+r_{i n}^{2}-2 r r_{i n} u_{i}+(z-h)^{2}}}{\left(r+r_{i n}\right)^{2}+(z-h)^{2}}\right], \frac{\left(r+r_{i n}\right)^{2}+(z-h)^{2}}{\left(r-r_{i n}\right)^{2}+(z-h)^{2}}\right] \\
& +\frac{\left(u_{i}^{2}-1\right)}{\sqrt{1-u_{i}^{2}}} \log \left[r_{\text {out }}-r u_{i}+\sqrt{r^{2}+r_{\text {out }}^{2}-2 r r_{\text {out }} u_{i}+(z-h)^{2}}\right] \\
& -\frac{\left(u_{i}^{2}-1\right)}{\sqrt{1-u_{i}^{2}}} \log \left[r_{\text {in }}-r u_{i}+\sqrt{r^{2}+r_{\text {in }}^{2}-2 r r_{\text {in }} u_{i}+(z-h)^{2}}\right]
\end{aligned}
$$

83

with :

$$
F_{1}\left(u_{i}, r, z\right)=\frac{1}{\sqrt{1-u_{i}^{2}}} \frac{2 r_{\text {out }}\left(1+u_{i}\right) \sqrt{\frac{r r_{\text {out }}\left(u_{i}-1\right)}{\left(r-r_{\text {out }}\right)^{2}+(z-h)^{2}}} \sqrt{\frac{r^{2}+r_{\text {out }}^{2}-2 r r_{\text {out }} u_{i}+(z-h)^{2}}{\left(r+r_{\text {out }}\right)^{2}+(z-h)^{2}}}}{\sqrt{\frac{r r_{\text {out }}\left(1+u_{i}\right)}{\left(r+r_{\text {out }}\right)^{2}+(z-h)^{2}}} \sqrt{r^{2}+r_{\text {out }}^{2}-2 r r_{\text {out }} u_{i}+(z-h)^{2}}}
$$

84

$$
F_{2}\left(u_{i}, r, z\right)=\frac{1}{\sqrt{1-u_{i}^{2}}} \frac{2 r_{i n}\left(1+u_{i}\right) \sqrt{\frac{r r_{i n}\left(u_{i}-1\right)}{\left(r-r_{i n}\right)^{2}+(z-h)^{2}}} \sqrt{\frac{r^{2}+r_{i n}^{2}-2 r r_{i n} u_{i}+(z-h)^{2}}{\left(r+r_{i n}\right)^{2}+(z-h)^{2}}}}{\sqrt{\frac{r r_{i n}\left(1+u_{i}\right)}{\left(r+r_{i n}\right)^{2}+(z-h)^{2}}} \sqrt{r^{2}+r_{i n}^{2}-2 r r_{i n} u_{i}+(z-h)^{2}}}
$$

85

86

87

$$
G_{1}(r, z)=\frac{\left(r-r_{i n}\right)^{2}+(z-h)^{2}}{2 r r_{i n}}
$$

${ }_{88}$ Equation (5) is valid for any observation point $M(r, \theta, z)$ with $\theta \neq \theta_{i}$ and $0 \leq \theta<2 \pi$. This expression 89 remains valid for ring permanent magnets, for which the angular width is $2 \pi\left(\theta_{2}-\theta_{1}=2 \pi\right)$. It leads 90 to the expression of the radial component already given by the authors for ring magnets [32]. Figure 3 91 represents the field radial component $H_{r}(r, \theta, z)$ versus the radial distance $r$. The used parameters are $92 h=3 \mathrm{~mm}, \theta_{1}=0 \mathrm{rad}, \theta_{2}=\frac{\pi}{2} \mathrm{rad}, \theta=0 \mathrm{rad}, r_{\text {in }}=25 \mathrm{~mm}, r_{\text {out }}=28 \mathrm{~mm}$.

93 3) Axial component $H_{z}(r, \theta, z)$ : The field axial component $H_{z}(r, \theta, z)$ created by the upper face is 94 given by (13).

$$
H_{z}(r, \theta, z)=\frac{\sigma^{*}}{4 \pi \mu_{0}}\left(\gamma\left(\theta, \theta_{2}\right)-\gamma\left(\theta, \theta_{1}\right)\right)
$$




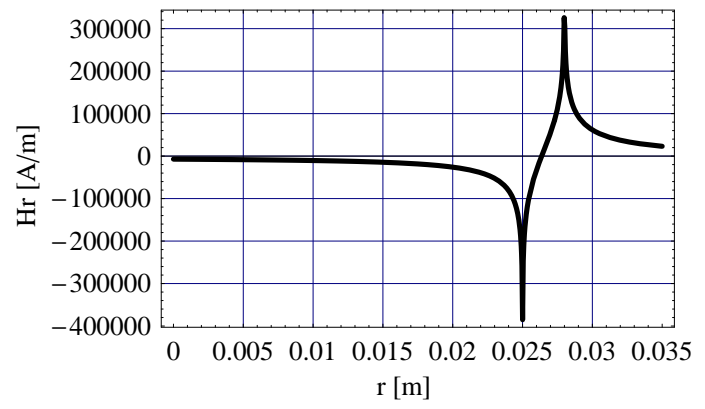

Fig. 3. Field radial component $H_{r}(r, \theta, z)$ versus the radial distance $r$ of the observation point; $h=3 m m, r_{i n}=25 m m$, $r_{\text {out }}=28 \mathrm{~mm}, \theta_{1}=0 \mathrm{rad}, \theta_{2}=\frac{\pi}{2} \operatorname{rad}, \theta=\frac{\pi}{2} \mathrm{rad}$

with

$$
\begin{aligned}
\gamma\left(\theta, \theta_{i}\right)= & h_{1} \eta_{1}\left(\theta, \theta_{i}\right) \boldsymbol{\Pi}^{*}\left[\frac{2\left(c_{1}+d_{1}\right) f}{2 c_{1} f-\sqrt{2} \sqrt{d_{1}^{2} f(-e+f)}}, i \sinh ^{-1}\left[\sqrt{\frac{-1}{c_{1}+d_{1}}} \sqrt{c_{1}-d_{1} \cos \left(\theta-\theta_{i}\right)}\right], \frac{c_{1}+d_{1}}{c_{1}-d_{1}}\right] \\
& +h_{2} \eta_{1}\left(\theta, \theta_{i}\right) \boldsymbol{\Pi}^{*}\left[\frac{2\left(c_{1}+d_{1}\right) f}{2 c_{1} f+\sqrt{2} \sqrt{d_{1}^{2} f(-e+f)}}, i \sinh ^{-1}\left[\sqrt{\frac{-1}{c_{1}+d_{1}}} \sqrt{c_{1}-d_{1} \cos \left(\theta-\theta_{i}\right)}\right], \frac{c_{1}+d_{1}}{c_{1}-d_{1}}\right] \\
& -h_{3} \eta_{2}\left(\theta, \theta_{i}\right) \boldsymbol{\Pi}^{*}\left[\frac{2\left(c_{2}+d_{2}\right) f}{2 c_{2} f-\sqrt{2} \sqrt{d_{2}^{2} f(-e+f)}}, i \sinh ^{-1}\left[\sqrt{\frac{-1}{c_{2}+d_{2}}} \sqrt{c_{2}-d_{2} \cos \left(\theta-\theta_{i}\right)}\right], \frac{c_{2}+d_{2}}{c_{2}-d_{2}}\right] \\
& -h_{4} \eta_{2}\left(\theta, \theta_{i}\right) \boldsymbol{\Pi}^{*}\left[\frac{2\left(c_{2}+d_{2}\right) f}{2 c_{2} f+\sqrt{2} \sqrt{d_{2}^{2} f(-e+f)}}, i \sinh ^{-1}\left[\sqrt{\frac{-1}{c_{2}+d_{2}}} \sqrt{c_{2}-d_{2} \cos \left(\theta-\theta_{i}\right)}\right], \frac{c_{2}+d_{2}}{c_{2}-d_{2}}\right]
\end{aligned}
$$

96 with

$$
\eta_{1}\left(\theta, \theta_{i}\right)=\frac{\left(-i \sqrt{\frac{d_{1}\left(-1+\cos \left(\theta-\theta_{i}\right)\right)}{c_{1}-d_{1}}} \sqrt{\frac{d_{1}\left(1+\cos \left(\theta-\theta_{i}\right)\right)}{c_{1}+d_{1}}} \frac{1}{\cos \left(\theta-\theta_{i}\right)}\right)}{\left(2 \sqrt{\frac{-1}{c_{1}+d_{1}}} \sqrt{d_{1}^{2} f\left(-e+d_{1}\right)}\left(d_{1}^{2}(e-f)\right)+2 c_{1}^{2} f\right)}
$$

97

$$
\eta_{2}\left(\theta, \theta_{i}\right)=\frac{\left(-i \sqrt{\frac{d_{2}\left(-1+\cos \left(\theta-\theta_{i}\right)\right)}{c_{2}-d_{2}}} \sqrt{\frac{d_{2}\left(1+\cos \left(\theta-\theta_{i}\right)\right)}{c_{2}+d_{2}}} \frac{1}{\cos \left(\theta-\theta_{i}\right)}\right)}{\left(2 \sqrt{\frac{-1}{c_{2}+d_{2}}} \sqrt{d_{2}^{2} f\left(-e+d_{2}\right)}\left(d_{2}^{2}(e-f)\right)+2 c_{2}^{2} f\right)}
$$

98

$$
h_{1}=2 a d_{1}\left(\sqrt{2} c_{1} f+\sqrt{d_{1}^{2} f(-e+f)}\right)+b_{1}\left(\sqrt{2} d_{1}^{2}(e-f)-2 c_{1} \sqrt{d_{1}^{2} f(-e+f)}\right)
$$




\begin{tabular}{|l|c|}
\hline Parameters & \\
\hline$a$ & $2(z-h)\left(r^{2}+(z-h)^{2}\right)$ \\
\hline$b_{1}$ & $2(z-h) r r_{\text {out }}$ \\
\hline$c_{1}$ & $r^{2}+r_{\text {out }}^{2}+(z-h)^{2}$ \\
\hline$d_{1}$ & $-2 r r_{\text {out }}$ \\
\hline$e$ & $-r^{2}-2(z-h)^{2}$ \\
\hline$f$ & $r^{2}$ \\
\hline$b_{2}$ & $2(z-h) r r_{\text {in }}$ \\
\hline$c_{2}$ & $r^{2}+r_{\text {in }}^{2}+(z-h)^{2}$ \\
\hline$d_{2}$ & $-2 r r_{\text {in }}$ \\
\hline
\end{tabular}

TABLE I

DEFINITION OF THE PARAMETERS USED IN (13)

$$
h_{2}=2 a d_{1}\left(-\sqrt{2} c_{1} f+\sqrt{d_{1}^{2} f(-e+f)}\right)+b_{1}\left(\sqrt{2} d_{1}^{2}(-e+f)-2 c_{1} \sqrt{d_{1}^{2} f(-e+f)}\right)
$$

$$
h_{3}=2 a d_{2}\left(\sqrt{2} c_{2} f+\sqrt{d_{2}^{2} f(-e+f)}\right)+b_{2}\left(\sqrt{2} d_{2}^{2}(e-f)-2 c_{2} \sqrt{d_{2}^{2} f(-e+f)}\right)
$$

$$
h_{4}=2 a d_{2}\left(-\sqrt{2} c_{2} f+\sqrt{d_{2}^{2} f(-e+f)}\right)+b_{2}\left(\sqrt{2} d_{2}^{2}(-e+f)-2 c_{2} \sqrt{d_{2}^{2} f(-e+f)}\right)
$$

where $\Pi^{*}[n, \phi, m]$ is given in terms of the incomplete elliptic integral of the third kind by (21).

$$
\boldsymbol{\Pi}^{*}[n, \phi, m]=\int_{0}^{\phi} \frac{1}{\left(1-n \sin (\theta)^{2}\right) \sqrt{1-m \sin (\theta)^{2}}} d \theta
$$

Although the result $H_{z}(r, \theta, z)$ is a real number, equation (14) contains the imaginary number $i\left(i^{2}=-1\right)$ because we did not succeed in obtaining a real expression for the axial component $H_{z}(r, \theta, z)$. The parameters used in (14) are defined in Table I. However, as the imaginary part is the consequence of numerical noise and nearly equals zero, when the expression (14) is used in symbolic mathematical tools such as Mathematica or Maple, the real part of $H z(r, \theta, z)$ only has to be considered. Equation (13) is valid for any observation point $M(r, \theta, z)$ with $\theta \neq \theta_{i}$ and $0 \leq \theta<2 \pi$. Here again, this expression remains valid for ring permanent magnets, i.e. when the angular width is $2 \pi\left(\theta_{2}-\theta_{1}=2 \pi\right)$. It also leads to the expression of the axial component already given by the authors for ring magnets [32]. Figure 4 represents the axial component $H_{z}(r, \theta, z)$ versus the radial distance $r$ of the observation point. The parameter values are $h=3 \mathrm{~mm}, \theta_{1}=0 \mathrm{rad}, \theta_{2}=\frac{\pi}{2} \mathrm{rad}, \theta=0 \mathrm{rad}, r_{\text {in }}=25 \mathrm{~mm}, r_{\text {out }}=28 \mathrm{~mm}$. 


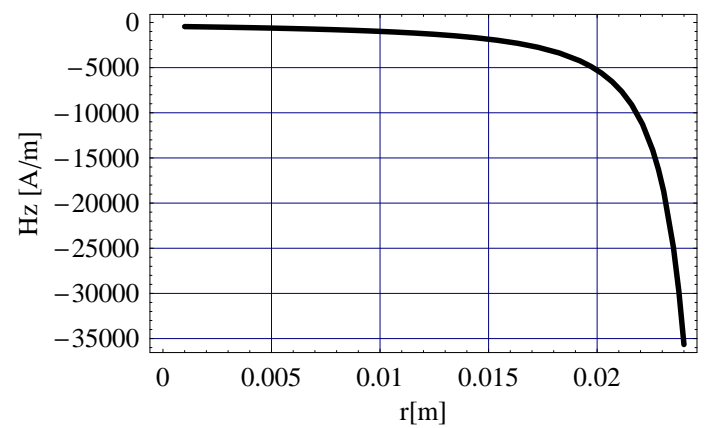

Fig. 4. Field axial component $H_{z}(r, \theta, z)$ versus the radial distance $r$ of the observation point; $h=3 m m, r_{i n}=25 m m$, $r_{\text {out }}=28 m m, \theta_{1}=0 \mathrm{rad}, \theta_{2}=\frac{\pi}{2} \mathrm{rad}, \theta=\frac{\pi}{2} \mathrm{rad}$

\section{RADIALLY MAGNETIZED TILES}

\section{A. Notation and geometry}

The geometry and its parameters are shown in Fig.(5). The axis $z$ is an axis of symmetry. Again, the coulombian model of permanent magnets is used. The permanent magnet ring sector is thus represented by two curved planes which correspond here to the inner and outer faces of the ring. The inner face is charged with a magnetic pole surface density $+\sigma^{*}$; the outer one is charged with the opposite magnetic pole surface density $-\sigma^{*}$. We only consider the inner face to simplify the analytical calculation. As stated previously, the total magnetic field can be calculated by the application of the linear superposition principle to both faces.

The magnetic pole volume density is not taken into account in this paper. This means that the total sum of all the charges in the model does not equal zero. Indeed, as the magnetization is radial, the magnetic pole surface density of the curved planes is uniform. The charge on the outer plane is thus greater than the charge on the inner plane, as the surfaces of these planes. The volume charge density, linked to the magnetization divergence, appears in fact to set the global charge to zero. If the radial width of the tile is small, which also means that the tile is thin, then the difference between the inner and outer plane surface is small, and so is the magnetic pole volume density: its neglecting is an acceptable approximation. This approximation becomes less and less valid when the thickness of the tile increases. This paper presents expressions for radially magnetized thin tiles.

Let us consider a point $P$ on the tile inner face. The magnetic field $\vec{H}$ created by the source point 


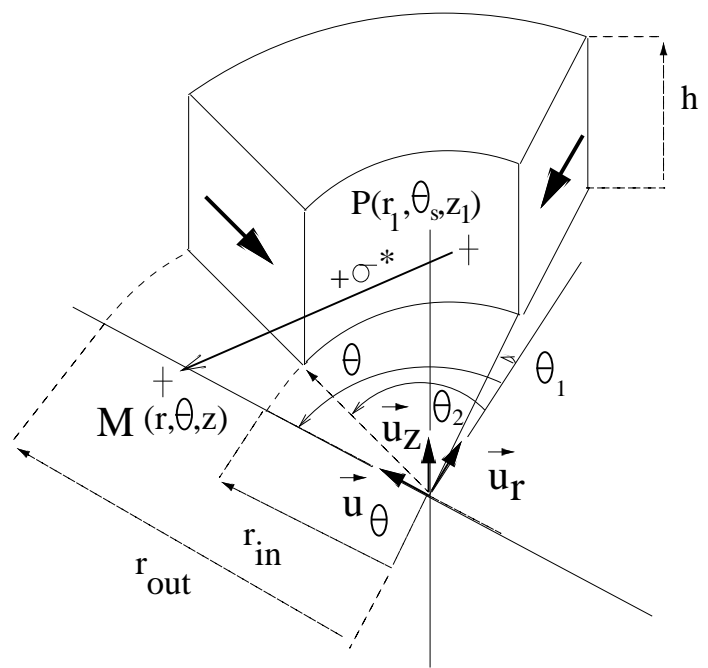

Fig. 5. Radially magnetized permanent magnet tile: parameter definition

$P\left(r, \theta_{s}, z\right)$ at any observation point $M(r, \theta, z)$ of the space is given by (22).

$$
\vec{H}(r, \theta, z)=\frac{\sigma^{*}}{4 \pi \mu_{0}} \int_{\theta_{1}}^{\theta_{2}} \int_{0}^{h} \frac{\overrightarrow{P M}}{|\overrightarrow{P M}|^{3}} r_{1} d z_{1} d \theta_{s}
$$

where $\mu_{0}$ is the magnetic permeability of the vacuum $\left(\mu_{0}=4 \pi \cdot 10^{-7} S I\right)$ and $\sigma^{*}$ is the fictitious magnetic pole surface density given in tesla . Equation (22) can be written as follows:

$$
\vec{H}(r, \theta, z)=\frac{\sigma^{*}}{4 \pi \mu_{0}} \int_{\theta_{1}}^{\theta_{2}} \int_{0}^{h} \frac{\left(r-r_{1} \cos \left(\theta-\theta_{s}\right)\right) \vec{u}_{r}-r_{1} \sin \left(\theta_{s}-\theta\right) \vec{u}_{\theta}+\left(z-z_{1}\right) \vec{u}_{z}}{\left(r_{1}^{2}+r^{2}+\left(z-z_{1}\right)^{2}-2 r_{1} r \cos \left(\theta-\theta_{s}\right)\right)^{\frac{3}{2}}} r_{1} d z_{1} d \theta_{s}
$$

B. Components along the three directions $\vec{u}_{r}, \vec{u}_{\theta}, \vec{u}_{z}$

The integration of (23) leads to the magnetic field components created by the inner face along the three axes defined $H_{r}(r, \theta, z), H_{\theta}(r, \theta, z), H_{z}(r, \theta, z)$.

1) Azimuthal component $H_{\theta}(r, \theta, z)$ : The field azimuthal component $H_{\theta}(r, \theta, z)$ is given by (24).

$$
H_{\theta}(r, \theta, z)=\frac{\sigma}{4 \pi \mu_{0}}\left(\beta\left(\theta, \theta_{1}\right)-\beta\left(\theta, \theta_{2}\right)\right)
$$




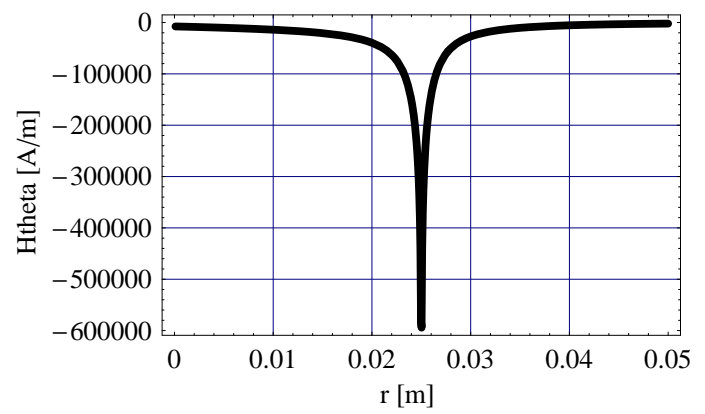

Fig. 6. Field azimuthal component $H_{\theta}(r, \theta, z)$ versus the radial distance $r$ of the observation point; $h=3 \mathrm{~mm}, r_{i n}=25 \mathrm{~mm}$, $r_{\text {out }}=28 \mathrm{~mm}, \theta_{1}=0 \mathrm{rad}, \theta_{2}=\frac{\pi}{2} \mathrm{rad}, \theta=0 \mathrm{rad}$

139

with

$$
\begin{aligned}
\beta\left(\theta, \theta_{i}\right)= & \frac{r_{i n}(b-z)}{r \sqrt{-(b-z)^{2}}} \arctan \left[\frac{\sqrt{r^{2}+r_{i n}^{2}+(b-z)^{2}-2 r r_{i n} \cos \left(\theta_{i}-\theta\right)}}{\sqrt{-(b-z)^{2}}}\right] \\
& -\frac{r_{i n}}{r} \tanh ^{-1}\left[\frac{\sqrt{r^{2}+r_{i n}^{2}+z^{2}-2 r r_{i n} \cos \left(\theta_{i}-\theta\right)}}{z}\right]
\end{aligned}
$$

Equation (24) is valid for any observation point $M(r, \theta, z)$ with $0 \leq \theta \leq 2 \pi$. Figure 6 represents the azimuthal component $H_{\theta}(r, \theta, z)$ versus the radial distance $r$ of the observation point. The parameters values are $h=3 \mathrm{~mm}, \theta_{1}=0, \theta_{2}=\frac{\pi}{2}, \theta=0, r_{\text {in }}=25 \mathrm{~mm}, r_{\text {out }}=28 \mathrm{~mm}$.

2) Radial component $H_{r}(r, \theta, z)$ : The radial component of the field $H_{r}(r, \theta, z)$ is given by (26).

$$
H_{r}(r, \theta, z)=\frac{\sigma^{*}}{4 \pi \mu_{0}}\left(\beta\left(u_{1}\right)-\beta\left(u_{2}\right)\right)
$$

with

$$
u_{i}=\cos \left(\theta-\theta_{i}\right)
$$

$$
\beta\left(u_{i}\right)=\left(\frac{2 i\left(1+u_{i}\right) \sqrt{\frac{d\left(-1+u_{i}\right)}{c+e_{1}+d u_{i}}}\left(-\left(a_{1} d+b_{1}\left(c+e_{1}\right)\right)\right) \mathbf{F}^{*}\left[i \sinh ^{-1}\left[\frac{\sqrt{-c+d-e_{1}}}{\sqrt{c+e_{1}+d u_{i}}}\right], \frac{c+d+e_{1}}{c-d+e_{1}}\right]}{d \sqrt{-c+d-e_{1}} e_{1} \sqrt{\frac{d\left(1+u_{i}\right)}{c+e_{1}+d u_{i}}} \sqrt{1-u_{i}^{2}}}\right)
$$




\begin{tabular}{|l|c|}
\hline Parameters & \\
\hline$a_{1}$ & $r_{i n} r z$ \\
\hline$b_{1}$ & $-r_{i n}^{2} z$ \\
\hline$c$ & $r^{2}+r_{i n}^{2}$ \\
\hline$d$ & $-2 r r_{i n}$ \\
\hline$e_{1} z^{2}$ \\
\hline$a_{2}$ & $-r_{i n} r(z-h)$ \\
\hline$b_{2}(z-h)$ \\
\hline$e_{2}$ & $r_{i n}^{2}(z-h)^{2}$ \\
\hline
\end{tabular}

TABLE II

DEFINITION OF THE PARAMETERS USED IN (26)

$$
\begin{aligned}
& +\left(\frac{2 i\left(1+u_{i}\right) \sqrt{\frac{d\left(-1+u_{i}\right)}{c+e_{1}+d u_{i}}}\left(b_{1} c-a_{1} d\right) \Pi^{*}\left[\frac{e_{1}}{c-d+e_{1}}, i \sinh ^{-1}\left[\frac{\sqrt{-c+d+e_{1}}}{c+e_{1}+d u_{i}}\right], \frac{c+d+e_{1}}{c-d+e_{1}}\right]}{d \sqrt{-c+d-e_{1}} e_{1} \sqrt{\frac{d\left(1+u_{i}\right)}{c+e_{1}+d u_{i}}} \sqrt{1-u_{i}^{2}}}\right) \\
& +\left(\frac{2 i\left(1+u_{i}\right) \sqrt{\frac{d\left(-1+u_{i}\right)}{c+e_{2}+d u_{i}}}\left(-\left(a_{2} d+b_{2}\left(c+e_{2}\right)\right)\right) \mathbf{F}^{*}\left[i \sinh ^{-1}\left[\frac{\sqrt{-c+d-e_{2}}}{\sqrt{c+e_{2}+d u_{i}}}\right], \frac{c+d+e_{2}}{c-d+e_{2}}\right]}{d \sqrt{-c+d-e_{2}} e_{2} \sqrt{\frac{d\left(1+u_{i}\right)}{c+e_{2}+d u_{i}}} \sqrt{1-u_{i}^{2}}}\right) \\
& +\left(\frac{2 i\left(1+u_{i}\right) \sqrt{\frac{d\left(-1+u_{i}\right)}{c+e_{2}+d u_{i}}}\left(b_{2} c-a_{2} d\right) \Pi^{*}\left[\frac{e_{2}}{c-d+e_{2}}, i \sinh ^{-1}\left[\frac{\sqrt{-c+d+e_{2}}}{c+e_{2}+d u_{i}}\right], \frac{c+d+e_{2}}{c-d+e_{2}}\right]}{d \sqrt{-c+d-e_{2}} e_{2} \sqrt{\frac{d\left(1+u_{i}\right)}{c+e_{2}+d u_{i}}} \sqrt{1-u_{i}^{2}}}\right)
\end{aligned}
$$

where $F^{*}[\phi, m]$ is defined by (31) and $\boldsymbol{\Pi}^{*}[n, \phi, m]$ is given by (21). Here again, Eq.(28) contains the imaginary number $i\left(i^{2}=-1\right)$ although the result $H_{r}(r, z)$ is a real number because we did not succeed in obtaining a real expression for the radial component $H_{r}(r, \theta, z)$. The parameters used in (28) are defined in Table II. Of course, when using the expression (28) in tools like Mathematica or Mapple, the imaginary part of $H_{r}(r, \theta, z)$ has to be dropped, as it only corresponds to numerical noise and nearly equals zero.

Equation (26) can be used to calculate the radial component of the magnetic field at any observation point $M(r, \theta, z)$ with $\theta \neq \theta_{i}$ and $0 \leq \theta<2 \pi$. When the tile angular width becomes $2 \pi\left(\theta_{2}-\theta_{1}=2 \pi\right)$, so for ring magnets, the expression becomes the one already given in a previous paper [32]. Figure 7 represents the field radial component $H_{r}(r, \theta, z)$ versus the radial distance $r$ of the observation point. The parameter values are $h=3 \mathrm{~mm}, \theta_{1}=0 \mathrm{rad}, \theta_{2}=\frac{\pi}{2} \mathrm{rad}, \theta=0 \mathrm{rad}, r_{\text {in }}=25 \mathrm{~mm}, r_{\text {out }}=28 \mathrm{~mm}$. 


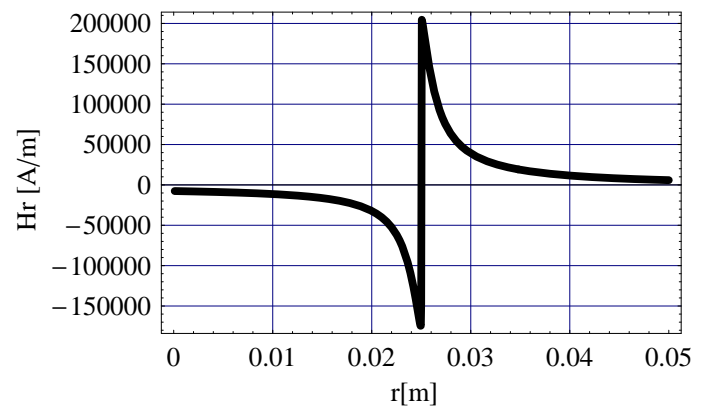

Fig. 7. Field radial component $H_{r}(r, \theta, z)$ versus the radial distance $r$ of the observation point; $h=3 \mathrm{~mm}, r_{i n}=25 \mathrm{~mm}$, $r_{\text {out }}=28 \mathrm{~mm}, \theta_{1}=0 \mathrm{rad}, \theta_{2}=\frac{\pi}{2} \operatorname{rad}, \theta=\frac{\pi}{2} \mathrm{rad}$

3) Axial component $H_{z}(r, \theta, z)$ : The field axial component $H_{z}(r, \theta, z)$ is given by (29):

$$
H_{z}(r, \theta, z)=\frac{\sigma^{*}}{4 \pi \mu_{0}}\left(\alpha\left(\theta, \theta_{2}\right)-\alpha\left(\theta, \theta_{1}\right)\right]
$$

with

$$
\begin{aligned}
\alpha\left(\theta, \theta_{i}\right)= & \frac{2 r_{i n}}{\left(r-r_{i n}\right)^{2}+(z-h)^{2}} \mathbf{F}^{*}\left[\frac{\theta-\theta_{i}}{2},-\frac{4 r r_{i n}}{\left(r-r_{i n}\right)^{2}+(z-h)^{2}}\right] \\
& -\frac{2 r_{i n}}{\left(r-r_{i n}\right)^{2}+z^{2}} \mathbf{F}^{*}\left[\frac{\theta-\theta_{i}}{2},-\frac{4 r r_{i n}}{\left(r-r_{i n}\right)^{2}+z^{2}}\right]
\end{aligned}
$$

where $\mathbf{F}^{*}[\phi, m]$ is given in terms of the elliptic integral of the first kind by (31).

$$
\mathbf{F}^{*}[\phi \mid m]=\int_{\theta=0}^{\theta=\phi} \frac{1}{\sqrt{1-m \sin (\theta)^{2}}} d \theta
$$

Equation (29) is valid for any observation point $M(r, \theta, z)$ with $0 \leq \theta \leq 2 \pi$. Figure 8 represents the field axial component $H_{z}(r, \theta, z)$ versus the radial distance $r$ of the observation point. The parameter values which are taken for the calculation are $h=3 \mathrm{~mm}, \theta_{1}=0 \mathrm{rad}, \theta_{2}=\frac{\pi}{2} \mathrm{rad}, \theta=0 \mathrm{rad}, r_{i n}=25 \mathrm{~mm}$, $r_{\text {out }}=28 \mathrm{~mm}$. 


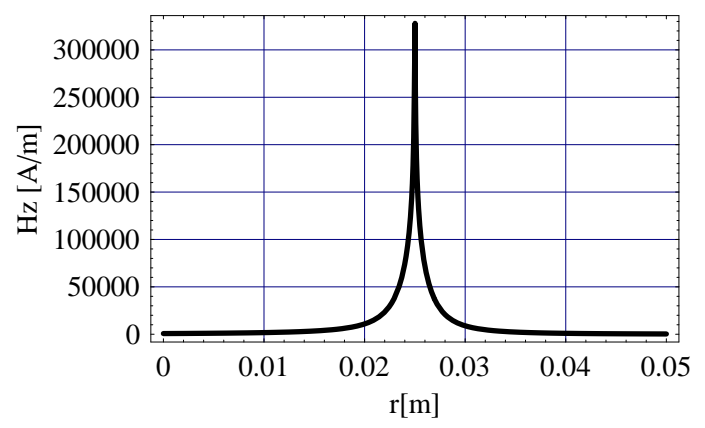

Fig. 8. Field axial component $H_{z}(r, \theta, z)$ versus the radial distance $r$ of the observation point; $h=3 \mathrm{~mm}, r_{i n}=25 \mathrm{~mm}$, $r_{\text {out }}=28 \mathrm{~mm}, \theta_{1}=0 \mathrm{rad}, \theta_{2}=\frac{\pi}{2} \operatorname{rad}, \theta=\frac{\pi}{2} \mathrm{rad}$

\section{CONCLUSION}

This paper gives the analytical expressions of the three components of the magnetic field created by tile permanent magnets whose magnetization is either radial or axial. When the tiles are axially magnetized, the magnetic field can be calculated using these expressions at any point in space. For radially magnetized tiles, the expressions given correspond to the field created by thin tiles, whose inner and outer radii are not too different, and can be used at any point of the space as well. The magnetic field is directly calculated, without the help of the magnetic potentials. The utility of such analytical expressions for the three components of the magnetic field created by tile permanent magnets lies in the fact that the related calculations have a low computational cost, especialy with regard to methods using finite elements or finite differences, and that they allow efficient parametric optimization studies of devices, which is very important for all applications.

The Mathematica files containing the analytical expressions used to calculate the three components of the magnetic field for axial and radial magnetizations are available line [33].

\section{REFERENCES}

[1] B. Azzerboni and G. Saraceno, "Three-dimensional calculation of the magnetic field created by current-carrying massive disks," IEEE Trans. Magn., vol. 34, no. 5, pp. 2601-2604, 1998.

[2] K. Kim, E. Levi, Z. Zabar, and L. Birenbaum, "Restoring force between two noncoaxial circular coils," IEEE Trans. Magn., vol. 32, no. 32, pp. 478-484, 1996. 
[3] K. Kim, E. Levi, Z. Zabar, and L. Birenbaum, "Mutual inductance of noncoaxial circular coils with constant current density," IEEE Trans. Magn., vol. 33, no. 32, pp. 4303-4309, 1997.

[4] O. M. Kwon, C. Surussavadee, M. V. K. Chari, S. Salon, and K. S. Vasubramaniam, "Analysis of the far field of permanent magnet motors and effects of geometric asymmetries and unbalance in magnet design," IEEE Trans. Magn., vol. 40, no. 3, pp. 435-442, 2004.

[5] J. P. Selvaggi, S. Salon, O. M. Kwon, and M. V. K. Chari, "Calculating the external magnetic field from permanent magnets in permanent-magnet motors - an alternative method," IEEE Trans. Magn., vol. 40, no. 5, pp. 3278-3285, 2004.

[6] S. Babic and C. Akyel, "Improvement in the analytical calculation of the magnetic field produced by permanent magnet rings," Progress in Electromagnetics Research C, vol. 5, pp. 71-82, 2008.

[7] S. Babic and C. Akyel, "An improvement in the calculation of the magetic field for an arbitrary geometry coil with rectangular cross section," International Journal of Numerical Modelling: Electronic Networks, Devices and Fields, vol. 18, pp. 493-504, November 2005.

[8] S. Babic and C. Akyel, "Magnetic force calculation between thin coaxial circular coils in air," IEEE Trans. Magn., vol. 44, no. 4, pp. 445-452, 2008.

[9] J. P. Selvaggi, S. Salon, O. M. Kwon, and M. V. K. Chari, "Computation of the three-dimensional magnetic field from solid permanent-magnet bipolar cylinders by employing toroidal harmonics," IEEE Trans. Magn., vol. 43, no. 10, pp. 3833-3839, 2007.

[10] J. Conway, "Inductance calculations for noncoaxial coils using bessel functions," IEEE Trans. Magn., vol. 43, no. 3, pp. 10231034, 2007.

[11] J. Conway, "Noncoaxial inductance calculations without the vector potential for axisymmetric coils and planar coils," IEEE Trans. Magn., vol. 44, no. 10, pp. 453-462, 2008.

[12] E. Perigo, R. Faria, and C. Motta, "General expressions for the magnetic flux density produced by axially magnetized toroidal permanent magnets," IEEE Trans. Magn., vol. 43, no. 10, pp. 3826-3832, 2008.

[13] Y. Zhilichev, "Calculation of magnetic field of tubular permanent magnet assemblies in cylindrical bipolar coordinates," IEEE Trans. Magn., vol. 43, no. 7, pp. 3189-3195, 2007.

[14] E. P. Furlani, S. Reznik, and A. Kroll, "A three-dimensonal field solution for radially polarized cylinders," IEEE Trans. Magn., vol. 31, no. 1, pp. 844-851, 1995 .

[15] E. P. Furlani, "A two-dimensional analysis for the coupling of magnetic gears," IEEE Trans. Magn., vol. 33, no. 3, pp. 23172321, 1997.

[16] E. P. Furlani, "Field analysis and optimization of ndfeb axial field permanent magnet motors," IEEE Trans. Magn., vol. 33, no. 5, pp. 3883-3885, 1997.

[17] H. L. Rakotoarison, J. P. Yonnet, and B. Delinchant, "Using coulombian approach for modeling scalar potential and magnetic field of a permanent magnet with radial polarization," IEEE Trans. Magn., vol. 43, no. 4, pp. 1261-1264, 2007.

[18] K. Halbach, "Design of permanent multiple magnets with oriented rec material," Nucl. Inst. Meth., vol. 169, pp. 1-10, 1980.

[19] J. Bird and A. Lipo, "Characteristics of an electrodynamic wheel using a 2-d steady-state model," IEEE Trans. Magn., vol. 43, no. 8, pp. 3396-3405, 2007. 
[20] J. Hilton and S. McMurry, "Halbach cylinders with improved field homogeneity and tailored gradient fields," IEEE Trans. Magn., vol. 43, no. 5, pp. 1898-1902, 2007.

[21] Z. Zhu, Z. Xia, and D. Howe, "Comparison of halbach magnetized brushless machines based on discrete magnet segments or a single ring magnet," IEEE Trans. Magn., vol. 38, no. 9, pp. 2997-2999, 2002.

[22] M. Aydin, Z. Zhu, T. Lipo, and D. Howe, "Minimization of cogging torque in axial-flux permanent-magnet machines: design concepts," IEEE Trans. Magn., vol. 43, no. 9, pp. 3614-3622, 2007.

[23] Y. Li, J. Zou, and Y. Lu, “Optimum design of magnet shape in permanent-magnet synchronous motors," IEEE Trans. Magn., vol. 39, no. 11, pp. 3523-4205, 2003.

[24] V. Lemarquand, J. F. Charpentier, and G. Lemarquand, "Nonsinusoidal torque of permanent-magnet couplings," IEEE Trans. Magn., vol. 35, no. 5, pp. 4200-4205, 1999.

[25] J. F. Charpentier and G. Lemarquand, "Calculation of ironless permanent magnet coupling using semi-numerical magnetic pole theory method," COMPEL, vol. 20, no. 1, pp. 72-89, 2001.

[26] C. Blache and G. Lemarquand, "High magnetic field gradients in flux confining permanent magnet structures," Journal of Magnetism and Magnetic Materials, vol. 104, pp. 1111-1112, 1992.

[27] G. Lemarquand, "Ironless loudspeakers," IEEE Trans. Magn., vol. 43, no. 8, pp. 3371-3374, 2007.

[28] R. Ravaud, G. Lemarquand, V. Lemarquand, and C. Depollier, "Ironless loudspeakers with ferrofluid seals," Archives of Acoustics, vol. 33, no. 4, pp. 3-10, 2008.

[29] R. Ravaud, G. Lemarquand, V. Lemarquand, and C. Depollier, "The three exact components of the magnetic field created by a radially magnetized tile permanent magnet.," Progress in Electromagnetics Research, PIER 88, pp. 307-319, 2008.

[30] R. Ravaud, G. Lemarquand, V. Lemarquand, and C. Depollier, "Discussion about the analytical calculation of the magnetic field created by permanent magnets.," Progress in Electromagnetics Research B, vol. 11, pp. 281-297, 2009.

[31] R. Ravaud, G. Lemarquand, V. Lemarquand, and C. Depollier, "Magnetic field produced by a tile permanent magnet whose polarization is both uniform and tangential," Progress in Electromagnetics Research B, vol. 12, 2009.

[32] R. Ravaud, G. Lemarquand, V. Lemarquand, and C. Depollier, "Analytical calculation of the magnetic field created by permanent-magnet rings," IEEE Trans. Magn., vol. 44, no. 8, pp. 1982-1989, 2008.

[33] http://www.univ-lemans.fr/ glemar. 